\title{
MicroRNA expression in esophageal squamous cell carcinoma: Novel diagnostic and prognostic biomarkers
}

\author{
YAN WANG $^{1}$, JINNAN ZHANG $^{2}$, WEI ZHAO ${ }^{1}$, DONGLIN WANG $^{3}$, WENDUAN MA $^{1}$, \\ SHENGTAO SHANG ${ }^{1}, \mathrm{CHAO}$ FENG $^{1}$ and HAIXIN YU ${ }^{1}$
}

\begin{abstract}
Departments of ${ }^{1}$ Thoracic Surgery and ${ }^{2}$ Neurosurgery, China-Japan Union Hospital of Jilin University, Changchun, Jilin 130033; ${ }^{3}$ Department of General Surgery, Green Garden Changchun City Hospital, Changchun, Jilin 130062, P.R. China
\end{abstract}

Received February 18, 2016; Accepted February 22, 2017

DOI: $10.3892 / \mathrm{mmr} .2017 .6479$

\begin{abstract}
The aim of the present study was to identify more effective molecular diagnostic biomarkers for esophageal squamous cell carcinoma (ESCC). The non-coding RNA profile GSE43732, generated from 238 paired frozen tissues from 119 patients, was analyzed. Raw data were preprocessed and the differentially expressed miRNAs were screened by limma package with $\log _{2}$ fold change $>2$. Prognosis-associated miRNAs were identified using receiver operating characteristic (ROC) and Kaplan-Meier (KM) curve analysis. miRNAs with an area under the ROC curve of $\geq 0.7$ were selected. miRNA target genes were identified from verification and predictive databases, and an miRNA regulatory network was constructed and visualized using Cytoscape software. Gene Ontology and pathway enrichment analyses of the target genes were performed using TargetMine. A total of 107 differentially expressed miRNAs, including 54 upregulated and 53 downregulated miRNAs, were obtained. The KM survival curves revealed that 44 miRNAs were significantly associated with prognosis. Furthermore, 9 upregulated and 3 downregulated miRNAs were obtained. Two upregulated miRNAs, hsa-miR-143-3p and hsa-miR-145-5p, and two downregulated miRNAs, hsa-miR-182-5p and hsa-miR-455-5p, were identified and demonstrated to be associated with prognosis in ESCC. In addition, 8 known and 245 predicted target genes of hsa-miR-455-5p were screened and the regulatory networks were constructed. Furthermore, these genes were functionally associated with macromolecule metabolic process and melanoma. In conclusion, two novel tumor suppressive miRNAs including miR-182-5p and miR-455-5p were identified. miR-455-5p in particular may be
\end{abstract}

Correspondence to: Dr Wei Zhao, Department of Thoracic Surgery, China-Japan Union Hospital of Jilin University, 126 Xiantai Street, Changchun, Jilin 130033, P.R. China

E-mail: ruweizhao08@163.com

Key words: esophageal squamous cell carcinoma, differentially expressed microRNA, microRNA regulatory network, target gene, prognosis involved in the regulation of ESCC. These miRNAs may be used to predict the prognosis of ESCC.

\section{Introduction}

Esophageal squamous cell carcinoma (ESCC), which begins in flat cells lining the esophagus, is a common esophageal malignant tumor globally, and particularly in China (1). Alcohol intake and tobacco smoking are major risk factors for ESCC $(2,3)$. It is possible to treat small localized ESCC $(<2 \mathrm{~cm})$ with surgery, while larger tumors may be slowed with chemotherapy and radiation therapy; however, as diagnosis is frequently late the outcomes tend to be relatively poor $(4,5)$. Therefore, multiple studies have been performed to study the pathogenesis of ESCC and to identify more effective molecular methods of diagnosis.

Lee etal (6) suggested thathereditary genetic polymorphisms of autophagy related 5 and collagen type IV $\alpha 3$ chain may be prognostic predictors for patients with ESCC. Elevated expression of AXL receptor tyrosine kinase in esophageal tumor tissues has been suggested to be associated with increased risk of death and recurrence of ESCC (7). Previous reports have identified genetic alternations of oncogenes and tumor suppressors in ESCC $(8,9)$, but the genetic and molecular basis for esophageal carcinogenesis remains largely unknown. Altered gene expression, which is the result of gene mutation or dramatic changes in gene regulation, is associated with a variety of diseases. MicroRNAs (miRNAs) act as oncogenes or tumor suppressors, mediating multiple gene expression in human cancers $(10,11)$. Previous studies demonstrated that in ESCC, tumor-suppressive miRNAs of miR-145, miR-133a, miR-133b (12) and miR-143 (13) inhibited tumor cell proliferation and invasion. MiR-155 (14), miR-21 (15) and miR-31 (16) act as oncogenes in ESCC. miRNA expression is correlated with carcinogenesis, and associated with prognosis and therapeutic outcomes. Guo et al (17) suggested that high expression of miR-103/107 was correlated with poor survival of patients with ESCC. miR-142-3p is a potential prognostic biomarker for ESCC (18). However, whether these miRNAs are efficient enough or whether it is possible to use them to evaluate prognosis in patients with ESCC remains unclear. Therefore, further studies are required to improve the survival rates of patients with ESCC. 
Using the non-coding RNA (miRNA) profile GSE43732, Chen et al (19) identified four miRNA signatures which had good prognostic value, and investigated the replicability and accuracy of these miRNAs as predictors. The present study used different methods to screen prognosis-associated miRNAs, and the results may provide novel predictable biomarkers and contribute to improved survival in patients with ESCC.

\section{Materials and methods}

Microarray data. The non-coding RNA profile GSE43732, which was contributed by Chen et al (19), was downloaded from the Gene Expression Omnibus database (20). Paired frozen tissues (cancer tissue and adjacent normal tissue) taken from 119 patients were assessed by microarray for miRNA expression analysis. This data was obtained based on the platform of GPL16543 Agilent-038166 cbc_human_miR18 (Cancer Institute and Hospital, Chinese Academy of Medical Sciences, Beijing, China).

Data preprocessing. Series Matrix Files containing normalized chip signals were transformed to gene symbols. The aggregate function in $\mathrm{R}$ package (bioconductor. org/packages $/ 2.1 / \mathrm{bioc} / \mathrm{html} /$ impute.html) was used to calculate the mean value when multiple probes were mapped to the same gene. The k-nearest neighbor method (21) from the impute package (22) in $\mathrm{R}$ was used with the default k-value of 10. Quantile normalization of miRNA expression profile was performed with the preprocessCore package (23) in R. The distribution of miRNA expression prior to and following normalization was visualized as a box plot.

Screening for differentially expressed miRNAs. Differentially expressed miRNAs were screened using the limma package (24) in R. The difference of mean expression level between two groups of samples was examined using the paired Student's $t$-test in the limma package. The $\mathrm{P}$-value was adjusted using the Benjamini-Hochberg method (25) to control the false discovery rate. Genes with $\log _{2}$ fold change $>2$ and adjusted $\mathrm{P}<0.05$ were considered to be differentially expressed miRNAs.

Identification of prognosis-related differentially expressed miRNAs. The high and low critical values of miRNA expression were derived based on the expression values of miRNAs and the survival time of patients using receiver operating characteristic (ROC) curve analysis. The ROC curves were generated and analyzed using Proc (26), KMsurv (27) and survival package (28) in R. Patients with lower and higher values compared with the critical values were divided into two groups. Subsequently, the significant variation of survival time between these two groups was examined using the Kaplan-Meier (KM) method and log rank test, with $\mathrm{P}<0.05$ considered to indicate a statistically significant difference.

Only miRNAs with an area under the curve (AUC) of $\geq 0.7$ were selected. They were subsequently screened again for miRNAs which were highly expressed in tumor tissues and associated with worse prognosis than others. Similarly, those miRNAs expressed at low levels in tumor tissues and associated with a worse prognosis were also identified.
Construction of the microRNA regulatory network for prognostic risk. Databases providing information on miRNA-target genes were used to identify the target genes of differentially expressed miRNAs. Verification databases included miRNecords (29) and MiRWalk (30), and predictive databases included miRanda (31), MirTarget2 (32), PicTar (33), PITA (34) and TargetScan (35). The identified target genes should be predicted by at least three of these databases. Target genes obtained from the two types of database were used to construct the miRNA-regulatory network, which was subsequently visualized using Cytoscape software (36).

Functional enrichment analysis of target genes. Gene Ontology (GO, http://www.geneontology.org/) analysis consisting of molecular function, cell component and biological process (BP), the Kyoto Encyclopedia of Genes and Genomes (KEGG) pathways (Reactome database, http://www. reactome.org/ and KEGG database, http://www.genome. $\mathrm{jp} / \mathrm{kegg} /$ ), and the Disease Ontology (BMC Genomics 10 Suppl 1:S6) enrichment analyses (37) for miRNAs-target genes were performed using the TargetMine (targetmine. nibio.go.jp) (38) online tool. The hypergeometric test within TargetMine for enrichment results was performed and the P-value was adjusted with the Holm-Bonferroni correction. An adjusted $\mathrm{P}<0.05$ was considered to indicate a statistically significant difference.

\section{Results}

Data normalization and differentially expressed miRNAs. Following normalization, a total of 726 miRNA expression values were obtained (Fig. 1). A total of 107 differentially expressed miRNAs, including 54 upregulated and 53 downregulated miRNAs, were obtained. The volcano plot of differentially expressed miRNAs is depicted in Fig. 2.

Prognosis related miRNAs. Critical values of the 107 differentially expressed miRNAs were determined. Kaplan-Meier (KM) curve analysis revealed that 44 miRNAs had significant effects on prognosis $(\mathrm{P}<0.05$; data not shown). Among them, 27 miRNAs were upregulated and 17 miRNAs were downregulated. Furthermore, 12 miRNAs, including 9 upregulated and 3 downregulated miRNAs, were screened with AUC $\geq 0.7$. In the end, hsa-miR-143-3p and hsa-miR-145-5p were revealed to be highly expressed in tumor tissues and were associated with prognosis (Fig. 3). Meanwhile, hsa-miR-182-5p and hsa-miR-455-5p, which were also associated with prognosis, were expressed at low levels in tumor tissues (Fig. 3).

Regulatory network of prognosis related miRNAs and target genes. A total of 8 target genes of hsa-miR-455-5p were obtained from the verification database, but not the other three miRNAs (hsa-miR-143-3p, hsa-miR-145-5p and miR-182-5p). The target genes were activating transcription factor 6 , interleukin 10 (IL10), toll-like receptor 4 (TLR4), activator of heat shock $90 \mathrm{kDa}$ protein ATPase homolog 1, scavenger receptor class B member I, prostaglandin-endoperoxide synthase 2, calreticulin and proopiomelanocortin. From the predicted databases, a total of 245 target genes including ring finger protein $(R N F) 44 / 111$, lysine demethylase (KDM) 3B/6A and 

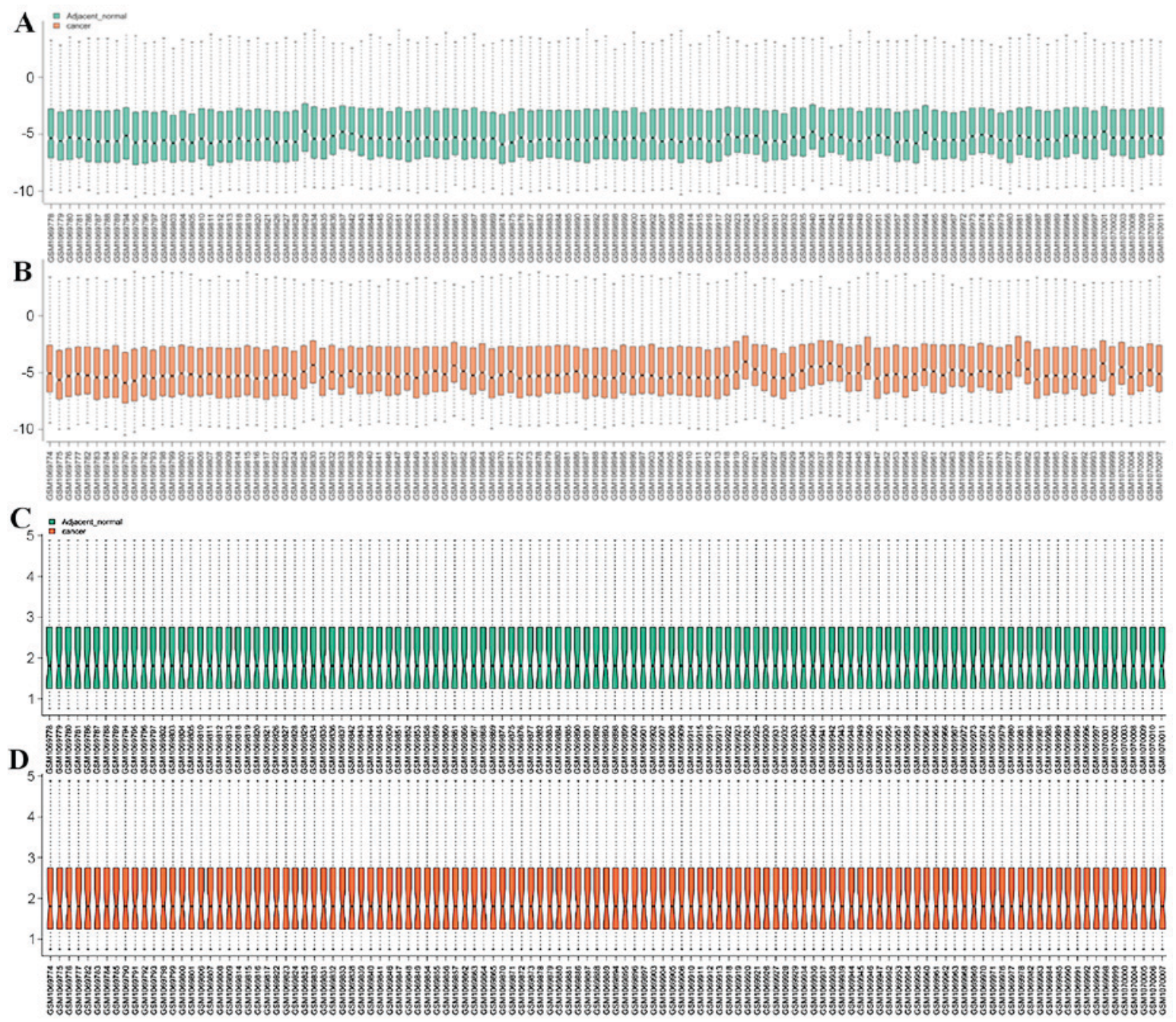

Figure 1. Box plots for each array for (A) adjacent normal tissue and (B) cancer tissue prior to normalization, and (C) adjacent normal tissue and (D) cancer tissue following normalization.

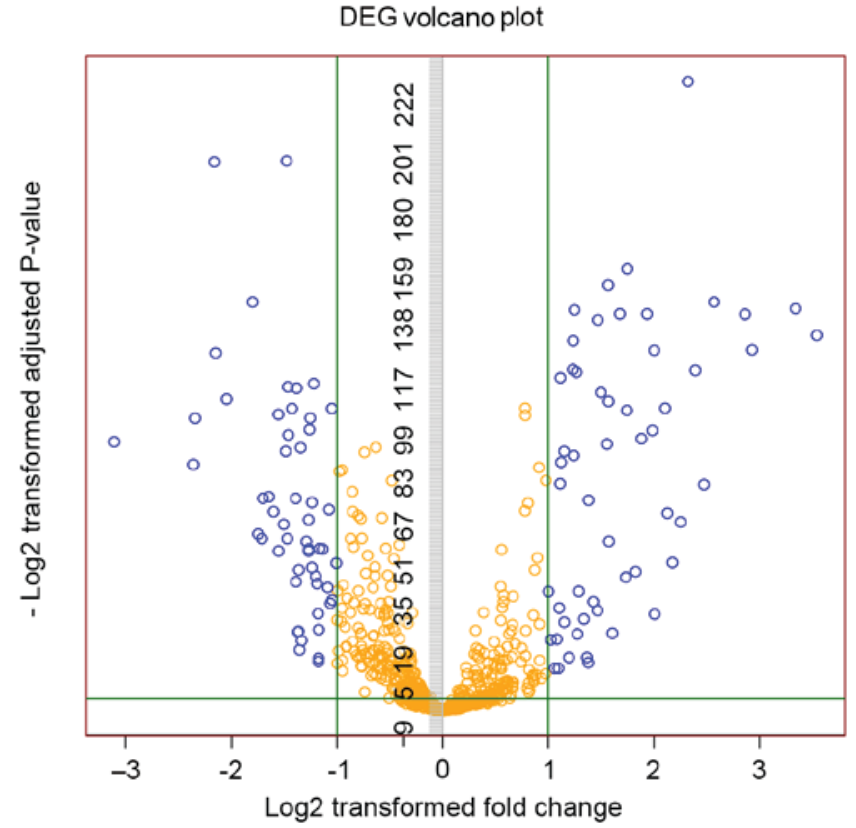

Figure 2. Volcano plot of differentially expressed miRNAs. The horizontal axis represents $\log _{2}$ fold change and the vertical axis represents- $\log _{10}$ (adjusted P-value). chromosome 15 open reading frame $26 / 41$, and the miRNA regulatory network is depicted in Fig. 4.

Functional enrichment analysis of target genes of miRNAs. No pathway was significantly enriched by the 253 target genes of hsa-miR-455-5p; however, two GO-BP terms were statistically significant (Table I). These were associated with the macromolecule metabolic process. The Disease Ontology enrichment results revealed that 15 target genes of hsa-miR-455-5p were enriched in term of melanoma (Table I).

\section{Discussion}

The poor prognosis of ESCC frequently results in severe adverse effects to patients. Novel, effective molecular markers for ESCC are, therefore, urgently required. In the present study, several miRNAs associated with prognosis of ESCC were discovered. miRNAs including miR-143-3p and miR-145-5p were upregulated, while miR-182-5p and miR-455-5p were downregulated in ESCC tissues. Previous research has demonstrated the involvement of miR-143-3p (39) and miR-145-5p in ESCC $(40,41)$, which were recorded in the MalaCards database; however, not the downregulated miRNAs miR-182-5p 

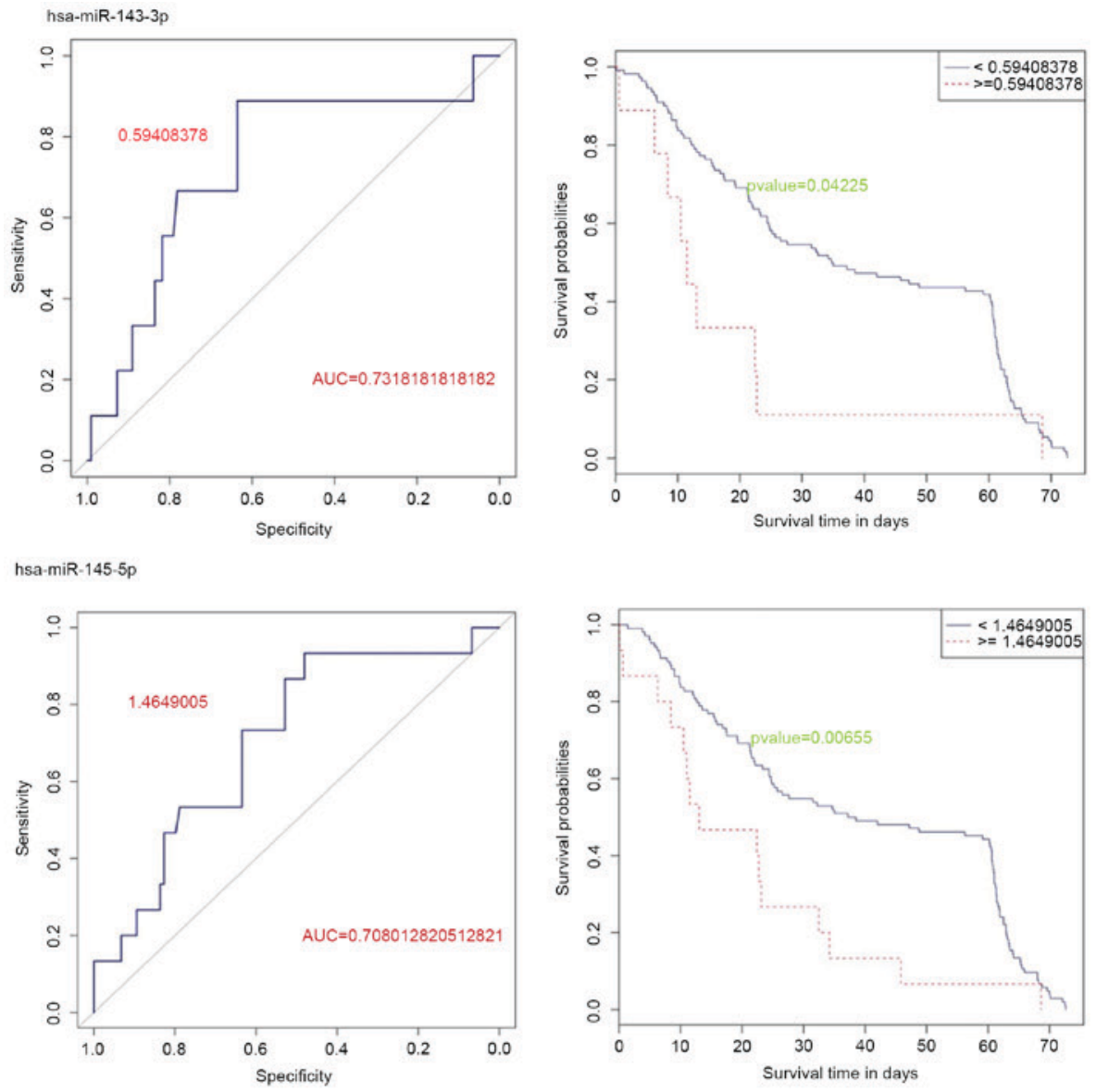

hsa-miR-182-5p
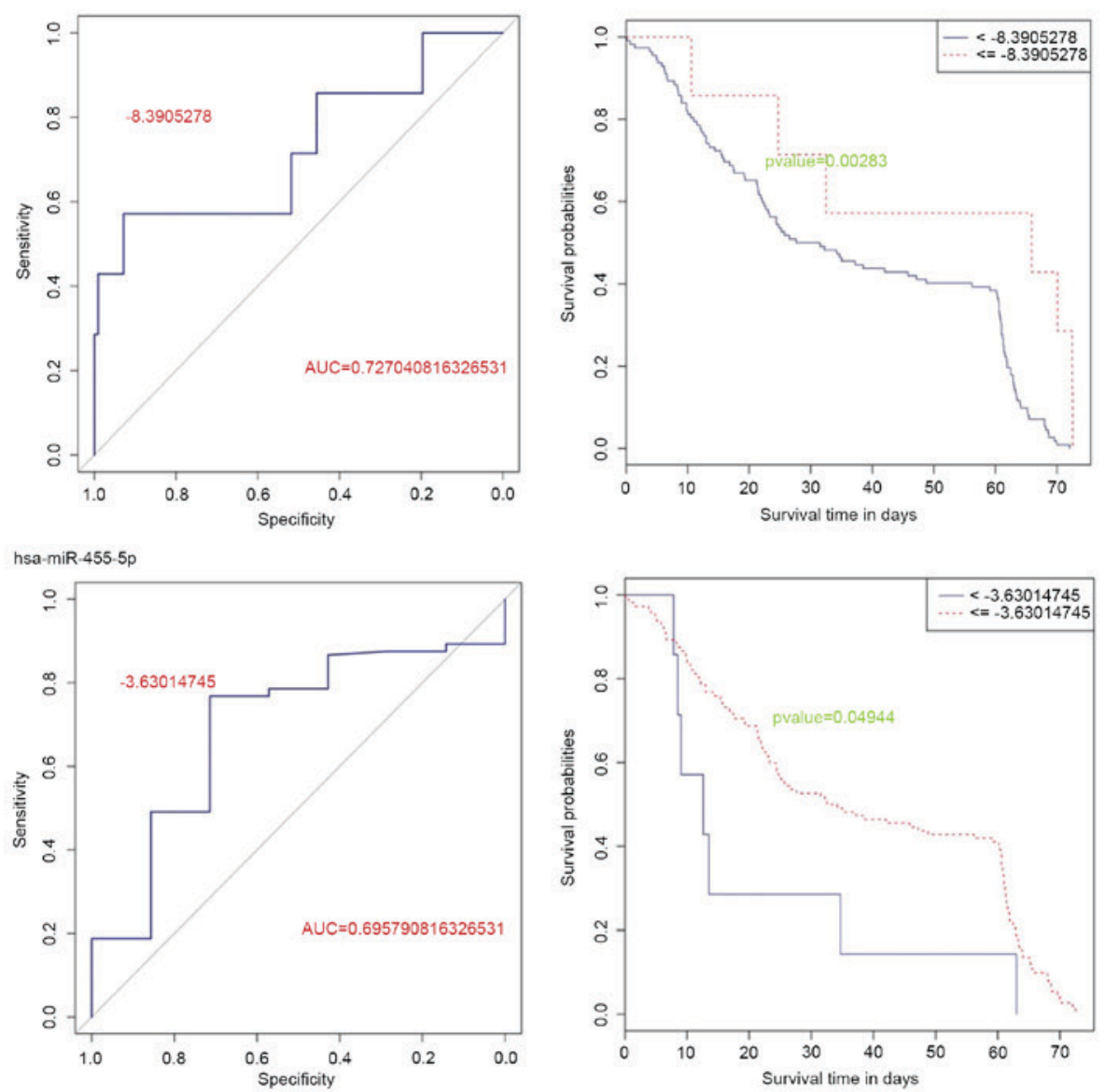

Figure 3. ROC curves (left) and Kaplan-Meier curves (right) of four miRs associated with good prognosis. The critical value is on the top left corner of the ROC curves. ROC, receiver operating curve; miR, microRNA. 
Table I. GO and DO enrichment analyses of target genes.

\begin{tabular}{llcc}
\hline Category & \multicolumn{1}{c}{ Term } & P-value & Count \\
\hline GO-BP & Macromolecule metabolic process [GO:0043170] & 0.00763 & 117 \\
GO-BP & Cellular macromolecule metabolic process [GO:0044260] & 0.00860 & 109 \\
DO term & Melanoma [DOID:1909] & 0.00215 & 15 \\
\hline
\end{tabular}

GO, Gene Ontology; DO, Disease Ontology; BP, biological process; count, the number of target genes enriched in this term.

A

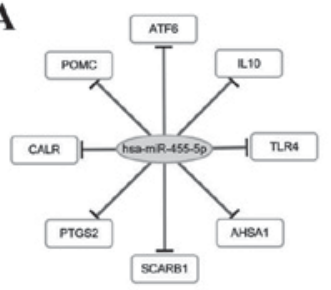

B

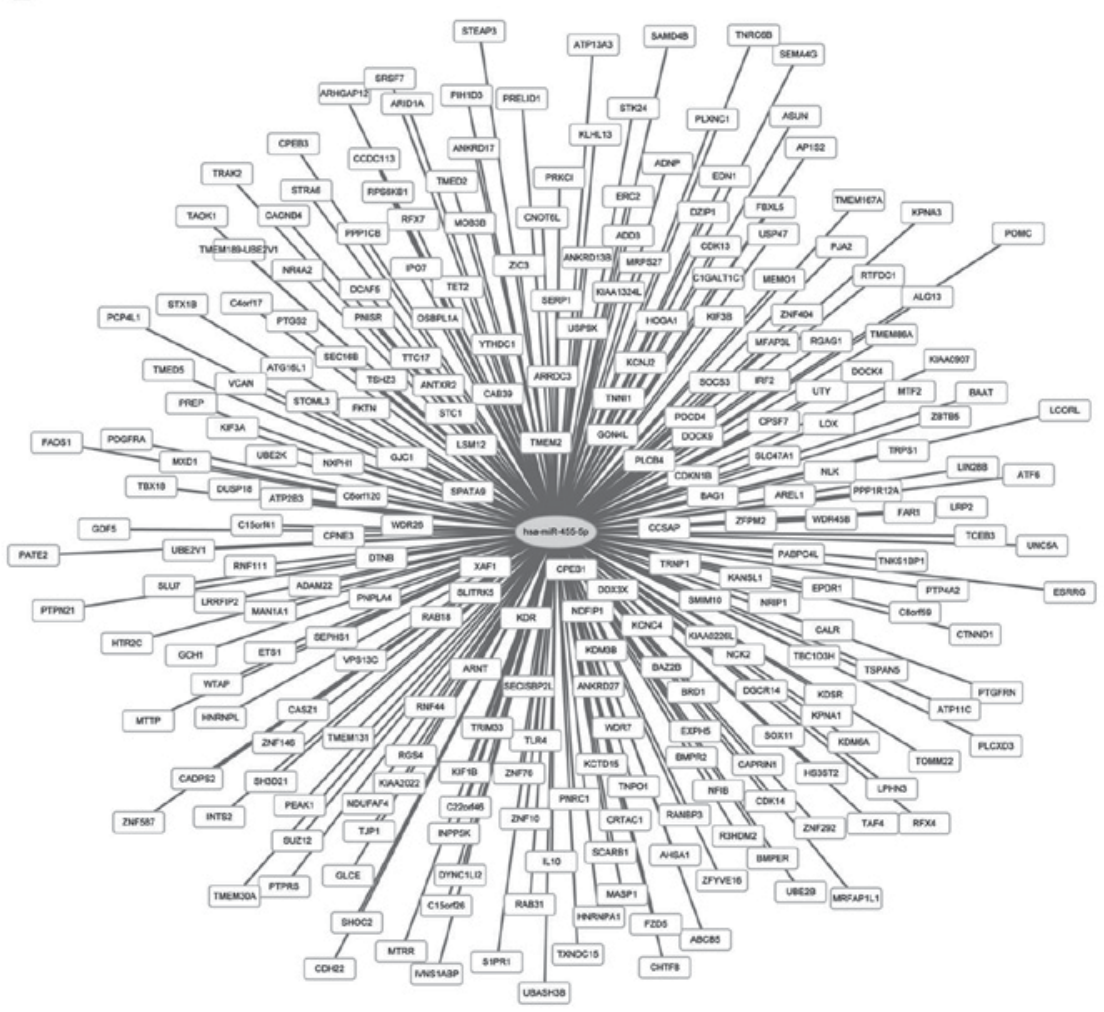

Figure 4. Regulatory network of hsa-miR-455-5p. (A) Regulatory network of eight verified target genes of hsa-miR-455-5p. (B) Regulatory network of 8 verified and 245 predicted genes targeted by hsa-miR-455-5p. hsa-miR-455-5p, human microRNA 455-5p.

and miR-455-5p. miR-182-5p and miR-455-5p may be novel miRNAs associated with ESCC.

The association between miR-182 and tumors has been widely studied. Segura et al (42) demonstrated that abnormal miR-182 expression represses forkhead box $\mathrm{O} 3$ and microphthalmia-associated transcription factors to promote melanoma metastasis, and contribute to renal cell carcinoma proliferation (43). miR-182 may be a prognostic marker for glioma progression and its expression was associated with patient survival (44). In the present study, miR-182-5p was revealed to be under expressed in tumor tissues and was associated with prognosis. Therefore, miR-182-5p may be a novel miRNA associated with the prognosis and survival rate of ESCC.

miR-455-5p was another downregulated miRNA identified in the present study. The expression pattern and its association with prognosis was similar to that of miR-182-5p.
Swingler et al (45) hypothesized that miR-455 may regulate apoptosis in certain mouse embryo tissue. Besides, decreased expression of miR-455-5p has been demonstrated to be correlated with vascular invasion and poor overall survival, and to be involved in endometrial serous adenocarcinomas progression (46). Based on these previous findings and the results of the present study, miR-455-5p may be a novel miRNA participating in the development and prognosis of ESCC. In addition, multiple target genes were identified. IL-10 (47) and TLR4 (48), which were overexpressed in the results of the present study, were demonstrated to be involved in ESCC. Among the predicted target genes, no genes recorded in the MalaCards database were associated with ESCC, but the homologous genes including RNF6 and $K D M 4 C$ were illustrated in this database. Furthermore, the Disease Ontology enrichment results demonstrated that the target genes were tumor-associated. In addition, $I L-10$ and 
$T L R 4$, as well as multiple other target genes of miR-455-5p, were revealed to be enriched in macromolecule biosynthesis and metabolism, which is associated with tumor initiation and progression $(49,50)$. Cellular metabolic perturbation is a crucial hallmark of cancer, and is associated with angiogenesis, evasion of apoptosis, metastasis and avoidance of immune detection (51). Macromolecule biosynthesis and metabolism are critical to supplying enough carbohydrates, proteins, lipids and nucleic acids to support the rapid cell division that occurs within a tumor (52). Therefore, miR-455-5p may be another novel miRNA that participates in the progression of ESCC and is associated with its prognosis.

In conclusion, in addition to the two known miRNAs (miR-143-3p and miR-145-5p), two novel tumor suppressor miRNAs, miR-182-5p and miR-455-5p, were demonstrated to be associated with ESCC progression. As multiple target genes of miR-455-5p were identified, it may be involved in complicated regulatory mechanisms and be involved in ESCC. Although there were no identified target genes for miR-182-5p, it may also be important in ESCC and warrants of further research. Whether the two miRNAs are effective targets for ESCC requires confirmation and further study in future experiments.

\section{References}

1. Lin D, Meng X, Xu L, Ding L, Garg M, Yang H, Liu L, Hao J, Wang M, Nagata y, et al: Comprehensive molecular characterization of esophageal squamous cell carcinoma. Cancer Res 74: 2225, 2014.

2. Lee CH, Lee JM, Wu DC, Hsu HK, Kao EL, Huang HL, Wang TN, Huang MC and Wu MT: Independent and combined effects of alcohol intake, tobacco smoking and betel quid chewing on the risk of esophageal cancer in Taiwan. Int J Cancer 113: 475-482, 2005.

3. Znaor A, Brennan P, Gajalakshmi V, Mathew A, Shanta V, Varghese C and Boffetta P: Independent and combined effects of tobacco smoking, chewing and alcohol drinking on the risk of oral, pharyngeal and esophageal cancers in Indian men. Int J Cancer 105: 681-686, 2003.

4. Stahl M, Mariette C, Haustermans K, Cervantes A and Arnold D; ESMO Guidelines Working Group: Oesophageal cancer: ESMO Clinical Practice Guidelines for diagnosis, treatment and follow-up. Ann Oncol 24 (Suppl 6): vi51-vi56, 2013.

5. Enzinger PC and Mayer RJ: Esophageal cancer. N Engl J Med 349: 2241-2252, 2003.

6. Lee JM, Yang PW, Chiang TH, Huang YC and Hsieh CY: The genetic polymorphisms of ATG5 and COL4A3 are associated with the prognosis of patients with esophageal squamous cell carcinoma. Cancer Res 74: 2859, 2014.

7. Yang PW, Hsieh MS, Huang YC, Chiang TH and Lee JM: AXL receptor tyrosine kinase is associated with the prognosis of patients with esophageal squamous cell carcinoma. Cancer Res 74: 4405, 2014.

8. Mandard AM, Hainaut P and Hollstein M: Genetic steps in the development of squamous cell carcinoma of the esophagus. Mutation Research/Reviews in Mutation Research 462: 335-342, 2000.

9. Ma S, Bao JY, Kwan PS, Chan YP, Tong CM, Fu L, Zhang N, Tong AH, Qin YR, Tsao SW, et al: Identification of PTK6, via RNA sequencing analysis, as a suppressor of esophageal squamous cell carcinoma. Gastroenterology 143: 675-686. e12, 2012.

10. Zhao Y, Schetter AJ, Yang GB, Nguyen G, Mathé EA, Li P, Cai H, Yu L, Liu F, Hang D, et al: microRNA and inflammatory gene expression as prognostic marker for overall survival in esophageal squamous cell carcinoma. Int J Cancer 132: 2901-2909, 2013.

11. Nohata N, Hanazawa T, Kikkawa N, Mutallip M, Sakurai D, Fujimura L, Kawakami K, Chiyomaru T, Yoshino H, Enokida H, et al: Tumor suppressive microRNA-375 regulates oncogene AEG-1/MTDH in head and neck squamous cell carcinoma (HNSCC). J Hum Genet 56: 595-601, 2011.
12. Kano M, Seki N, Kikkawa N, Fujimura L, Hoshino I, Akutsu Y, Chiyomaru T, Enokida H, Nakagawa $M$ and Matsubara $H$ : miR-145, miR-133a and miR-133b: Tumor-suppressive miRNAs target FSCN1 in esophageal squamous cell carcinoma. Int J Cancer 127: 2804-2814, 2010.

13. Ni Y, Meng L, Wang L, Dong W, Shen H, Wang G, Liu Q and Du J: MicroRNA-143 functions as a tumor suppressor in human esophageal squamous cell carcinoma. Gene 517: 197-204, 2013.

14. Zhang J, Cheng C, Yuan X, He JT, Pan QH and Sun FY: microRNA-155 acts as an oncogene by targeting the tumor protein 53-induced nuclear protein 1 in esophageal squamous cell carcinoma. Int J Clin Exp Pathol 7: 602, 2014.

15. Ma WJ, Lv GD, Tuersun A, Liu Q, Liu H, Zheng ST, Huang CG, Feng JG, Wang X, Lin RY, et al: Role of microRNA-21 and effect on PTEN in Kazakh's esophageal squamous cell carcinoma. Mol Biol Rep 38: 3253-3260, 2011.

16. Zhang T, Wang Q, Zhao D, Cui Y, Cao B, Guo L and Lu SH: The oncogenetic role of microRNA-31 as a potential biomarker in oesophageal squamous cell carcinoma. Clin Sci (Lond) 121: 437-447, 2011.

17. Guo Y, Chen Z, Zhang L, Zhou F, Shi S, Feng X, Li B, Meng X, Ma X, Luo M, et al: Distinctive microRNA profiles relating to patient survival in esophageal squamous cell carcinoma. Cancer Res 68: 26-33, 2008

18. Lin RJ, Xiao DW, Liao LD, Chen T, Xie ZF, Huang WZ, Wang WS, Jiang TF, Wu BL, Li EM and Xu LY: MiR-142-3p as a potential prognostic biomarker for esophageal squamous cell carcinoma. J Surg Oncol 105: 175-182, 2012.

19. Chen Z, Li J, Tian L, Zhou C, Gao Y, Zhou F, Shi S, Feng X, Sun N, Yao R, et al: MiRNA expression profile reveals a prognostic signature for esophageal squamous cell carcinoma. Cancer Lett 350: 34-42, 2014.

20. Barrett T and Edgar R: Gene expression omnibus: Microarray data storage, submission, retrieval, and analysis. Methods Enzymol 411: 352-369, 2006.

21. Altman NS: An introduction to kernel and nearest-neighbor nonparametric regression. The American Statistician 46: 175-185, 1992.

22. Hastie T, Tibshirani R, Narasimhan B and Chu G: Impute: Imputation for microarray data. R package version, 2001.

23. Bolstad B: PreprocessCore: A collection of pre-processing functions. R package version 1.20.0, 2013.

24. Smyth GK: Limma: linear models for microarray data. In: Bioinformatics and computational biology solutions using R and Bioconductor. Springer, pp397-420, 2005.

25. Benjamini Y and Hochberg Y: Controlling the false discovery rate: A practical and powerful approach to multiple testing. Journal of the Royal Statistical Society. Series B (Methodological) 57: 289-300, 1995.

26. Robin X, Turck N, Hainard A, Tiberti N, Lisacek F, Sanchez JC and Müller M: pROC: An open-source package for R and S+ to analyze and compare ROC curves. BMC Bioinformatics 12: 77, 2011.

27. KMsurv: Data sets from Klein and Moeschberger (1997), Survival Analysis. R package version 0.1-5, 2012. https:// cran.r-project.org/web/packages/KMsurv/KMsurv.pdf Accessed Febuary 19, 2015.

28. Therneau TM and Grambsch PM: Modeling Survival Data: Extending the Cox model. New York, NY, Springer, 2000.

29. Xiao F, Zuo Z, Cai G, Kang S, Gao X and Li T: miRecords: An integrated resource for microRNA-target interactions. Nucleic Acids Res 37: D105-D110, 2009.

30. Dweep H, Sticht C, Pandey P and Gretz N: miRWalk-database: Prediction of possible miRNA binding sites by 'walking' the genes of three genomes. J Biomed Inform 44: 839-847, 2011.

31. Enright AJ, John B, Gaul U, Tuschl T, Sander C and Marks DS: MicroRNA targets in Drosophila. Genome Biol 5: R1, 2003.

32. Wang $X$ and El Naqa IM: Prediction of both conserved and nonconserved microRNA targets in animals. Bioinformatics 24: 325-332, 2008.

33. Krek A, Grün D, Poy MN, Wolf R, Rosenberg L, Epstein EJ, MacMenamin P, da Piedade I, Gunsalus KC, Stoffel M and Rajewsky N: Combinatorial microRNA target predictions. Nat Genet 37: 495-500, 2005.

34. Kertesz M, Iovino N, Unnerstall U, Gaul U and Segal E: The role of site accessibility in microRNA target recognition. Nat Genet 39: 1278-1284, 2007

35. Lewis BP, Shih IH, Jones-Rhoades MW, Bartel DP and Burge CB: Prediction of mammalian microRNA targets. Cell 115: 787-798, 2003. 
36. Shannon P, Markiel A, Ozier O, Baliga NS, Wang JT, Ramage D, Amin N, Schwikowski B and Ideker T: Cytoscape: A software environment for integrated models of biomolecular interaction networks. Genome Res 13: 2498-2504, 2003.

37. Schriml LM, Arze C, Nadendla S, Chang YW, Mazaitis M, Felix V, Feng G and Kibbe WA: Disease Ontology: A backbone for disease semantic integration. Nucleic Acids Res 40: D940-D946, 2012

38. Chen YA, Tripathi LP and Mizuguchi K: TargetMine, an integrated data warehouse for candidate gene prioritisation and target discovery. PLoS One 6: e17844, 2011.

39. Wu XL, Cheng B, Li PY, Huang HJ, Zhao Q, Dan ZL, Tian DA and Zhang P: MicroRNA-143 suppresses gastric cancer cell growth and induces apoptosis by targeting COX-2. World $\mathrm{J}$ Gastroenterol 19: 7758-7765, 2013.

40. Wu BL, Xu LY, Du ZP, Liao LD, Zhang HF, Huang Q, Fang GQ and Li EM: MiRNA profile in esophageal squamous cell carcinoma: Downregulation of miR-143 and miR-145. World J Gastroenterol 17: 79-88, 2011.

41. Liu R, Liao J, Yang M, Sheng J, Yang H, Wang Y, Pan E, Guo W, Pu Y, Kim SJ and Yin L: The cluster of miR-143 and miR-145 affects the risk for esophageal squamous cell carcinoma through co-regulating fascin homolog 1. PLoS One 7: e33987, 2012.

42. Segura MF, Hanniford D, Menendez S, Reavie L, Zou X, Alvarez-Diaz S, Zakrzewski J, Blochin E, Rose A, Bogunovic D, et al: Aberrant miR-182 expression promotes melanoma metastasis by repressing FOXO3 and microphthalmia-associated transcription factor. Proc Natl Acad Sci USA 106: 1814-1819, 2009.

43. Xu X, Wu J, Li S, Hu Z, Xu X, Zhu Y, Liang Z, Wang X, Lin Y, Mao Y, et al: Downregulation of microRNA-182-5p contributes to renal cell carcinoma proliferation via activating the AKT/FOXO3a signaling pathway. Mol Cancer 13: 109, 2014

44. Jiang L, Mao P, Song L, Wu J, Huang J, Lin C, Yuan J, Qu L, Cheng SY and Li J: miR-182 as a prognostic marker for glioma progression and patient survival. Am J Pathol 177: 29-38, 2010
45. Swingler TE, Wheeler G, Carmont V, Elliott HR, Barter MJ, Abu-Elmagd M, Donell ST, Boot-Handford RP, Hajihosseini MK, Münsterberg A, et al: The expression and function of microRNAs in chondrogenesis and osteoarthritis. Arthritis Rheum 64: 1909-1919, 2012.

46. Hiroki E, Akahira J, Suzuki F, Nagase S, Ito K, Suzuki T, Sasano $\mathrm{H}$ and Yaegashi N: Changes in microRNA expression levels correlate with clinicopathological features and prognoses in endometrial serous adenocarcinomas. Cancer Sci 101: 241-249, 2010.

47. Gholamin M, Moaven O, Memar B, Farshchian M, Naseh H, Malekzadeh R, Sotoudeh M, Rajabi-Mashhadi MT, Forghani MN, Farrokhi $\mathrm{F}$ and Abbaszadegan MR: Overexpression and interactions of interleukin-10, transforming growth factor beta, and vascular endothelial growth factor in esophageal squamous cell carcinoma. World J Surg 33: 1439-1445, 2009.

48. Sheyhidin I, Nabi G, Hasim A, Zhang RP, Ainiwaer J, Ma H and Wang H: Overexpression of TLR3, TLR4, TLR7 and TLR9 in esophageal squamous cell carcinoma. World J Gastroenterol 17: 3745-3751, 2011.

49. Baxter LT and Jain RK: Transport of fluid and macromolecules in tumors: III. Role of binding and metabolism. Microvasc Res 41: 5-23, 1991.

50. Hsu PP and Sabatini DM: Cancer cell metabolism: Warburg and beyond. Cell 134: 703-707, 2008

51. Masoudi-Nejad A and Asgari Y: Metabolic cancer biology: Structural-based analysis of cancer as a metabolic disease, new sights and opportunities for disease treatment. Journal 30: 21-29, 2015.

52. Ward PS and Thompson CB: Metabolic reprogramming: A cancer hallmark even warburg did not anticipate. Cancer cell 21: 297-308, 2012. 